\title{
Identification of Differentially Expressed Genes in Pituitary Adenomas by Integrating Analysis of Microarray Data
}

\author{
Peng Zhao, ${ }^{1}$ Wei Hu, ${ }^{2}$ Hongyun Wang, ${ }^{3}$ Shengyuan Yu, ${ }^{3}$ Chuzhong Li, ${ }^{3}$ Jiwei Bai, \\ Songbai Gui, ${ }^{1}$ and Yazhuo Zhang ${ }^{3}$ \\ ${ }^{1}$ Department of Neurosurgery, Beijing Tiantan Hospital, Capital Medical University, Beijing, China \\ ${ }^{2}$ Department of Cardiology, Beijing Chuiyangliu Hospital, Beijing, China \\ ${ }^{3}$ Beijing Neurosurgical Institute, Center of Brain Tumor, Beijing Institute for Brain Disorders, \\ Capital Medical University, Beijing, China
}

Correspondence should be addressed to Yazhuo Zhang; zhang-yazhuo@163.com

Received 22 September 2014; Revised 16 December 2014; Accepted 16 December 2014

Academic Editor: Maria L. Dufau

Copyright (C) 2015 Peng Zhao et al. This is an open access article distributed under the Creative Commons Attribution License, which permits unrestricted use, distribution, and reproduction in any medium, provided the original work is properly cited.

\begin{abstract}
Pituitary adenomas, monoclonal in origin, are the most common intracranial neoplasms. Altered gene expression as well as somatic mutations is detected frequently in pituitary adenomas. The purpose of this study was to detect differentially expressed genes (DEGs) and biological processes during tumor formation of pituitary adenomas. We performed an integrated analysis of publicly available GEO datasets of pituitary adenomas to identify DEGs between pituitary adenomas and normal control (NC) tissues. Gene function analysis including Gene Ontology (GO), Kyoto Encyclopedia of Genes and Genomes (KEGG) pathway enrichment analysis, and protein-protein interaction (PPI) networks analysis was conducted to interpret the biological role of those DEGs. In this study we detected 3994 DEGs (2043 upregulated and 1951 downregulated) in pituitary adenoma through an integrated analysis of 5 different microarray datasets. Gene function analysis revealed that the functions of those DEGs were highly correlated with the development of pituitary adenoma. This integrated analysis of microarray data identified some genes and pathways associated with pituitary adenoma, which may help to understand the pathology underlying pituitary adenoma and contribute to the successful identification of therapeutic targets for pituitary adenoma.
\end{abstract}

\section{Introduction}

Pituitary adenomas account for $10-15 \%$ of all intracranial neoplasms. Most pituitary adenomas are benign, although they may cause significant morbidity through mass effects and/or the improper secretion of pituitary hormones, indicating that the development of pituitary adenomas is a complex multistep process. Pituitary adenomas usually occur sporadically and are grouped into functioning and nonfunctioning adenomas (NFAs) according to hormonal status and further subdivided into microadenomas $(<1 \mathrm{~cm})$ and macroadenomas $(\geq 1 \mathrm{~cm})$ based on tumor size [1].

Despite massive research, the pathogenesis of pituitary adenomas still remains unclear. However, advances in molecular biology such as microarray technique enable the identification of new genes associated with pituitary tumor genesis. The microarray technique, which allows the simultaneous analysis of thousands of genes at the transcript expression level in a single experiment [2], has greatly facilitated the investigation of gene expression differences between normal pituitary and pituitary adenomas. Recently, researchers have used this powerful technique to compare gene expression between normal pituitary tissues and pituitary adenomas of different origins and have also identified many genes associated with certain tumor types [3-6]. However, there are inconsistencies among these studies due to limitations of different sample sources, microarray platforms, and analysis techniques [7]. Towards this end, we performed a systematic integration of gene expression data from multiple sources, to increase statistical power for detecting differentially expressed genes (DEGs) $[8,9]$. Now in this study we use this method to identify DEGs and biological processes associated with pituitary adenomas to provide some insights 
TABLE 1: Characteristics of the individual studies.

\begin{tabular}{|c|c|c|c|c|}
\hline GEO ID & Author & Platform & Samples $(\mathrm{N}: \mathrm{P})$ & Year \\
\hline GSE51618 & Feng J & GPL6480 Agilent-014850 4x44K G4112F & $3: 7$ & 2013 \\
\hline GSE46311 & Lekva T & GPL6244 Affymetrix Human Gene 1.0 ST Array & $0: 16$ & 2013 \\
\hline GSE36314 & Oyesiku [10] & GPL8300 Affymetrix Human Genome U95 Version 2 Array & $3: 4$ & 2012 \\
\hline GSE22812 & Wierinckx et al. [11] & GPL2895 GE Healthcare/Amersham Biosciences CodeLink Bioarray [11] & $0: 13$ & 2011 \\
\hline GSE4237 & Hussaini IM & GPL570 Affymetrix Human Genome U133 Plus 2.0 Array & $6: 4$ & 2006 \\
\hline
\end{tabular}

into molecular mechanisms underlying the pathogenesis of pituitary adenomas and many guided further therapies for this disease.

\section{Material and Methods}

2.1. Identification of Eligible Gene Expression Datasets. Expression profiling studies of pituitary adenomas were identified by searching the Gene Expression Omnibus database (GEO, http://www.ncbi.nlm.nih.gov/geo/) [12]. We only collected original experimental articles that analyzed gene expression profiling between pituitary adenoma and normal control (NC) tissues. Nonhuman studies, review articles, and integrated analysis of expression profiles were excluded.

2.2. Data Preprocessing. Normalization is very important to compare multiple microarray datasets accurately. The heterogeneity of multiple datasets resulted from different platforms, and clinical samples may make it difficult to compare the results directly. Consequently a global normalization approach should be included to minimize the heterogeneity. For this purpose, we first preprocessed the raw microarray data of each study by $\log 2$ transformation, then the Z-score transformation was applied for calculation of expression intensities of each probe, and Z-scores were calculated following the formula

$$
\mathrm{Z} \text { score }=\frac{x_{i}-\bar{x}}{\delta}
$$

where $x_{i}$ indicates raw intensity data for each gene; $\bar{x}$ indicates the average intensity of the gene in a single experiment, and $\delta$ indicates standard deviation (SD) of all the measured intensities.

2.3. Statistical Analysis. The significance analysis of microarray (SAM) software was used to determine the DEGs between pituitary adenoma and NC tissues. Gene specific $t$-tests were carried out, outputting a "relative difference" score or $d$ value which was defined as the average expression change of each gene from different expression levels to the SD of measurements. The genes with at least 1.5-fold change and a false discovery rate (FDR) less than 0.05 were selected as DEGs [13].

2.4. Functional Annotation of DEGs. To interpret the biological functions of the DEGs, we performed Gene Ontology
(GO) enrichment analysis to explore functional distribution of DEGs in pituitary adenoma. GO provides a common descriptive framework and functional annotation of the gene sets data. Furthermore we also performed Kyoto Encyclopedia of Genes and Genomes (KEGG) pathway enrichment analysis for DEGs to find important pathways involved in pituitary adenoma. KEGG pathway database is a recognized and comprehensive database including all kinds of biochemistry pathways [14]. The online based software GENECODIS was utilized in this analysis [15].

2.5. PPI Network Construction. The protein-protein interactions (PPIs) analysis was conducted to investigate the functions of proteins at the molecular level [16]. The identification of protein interactions in a genome-wide scale is important to uncover the cellular regulation mechanisms [17]. Biological General Repository for Interaction Datasets (BioGRID) (http://thebiogrid.org/) was used to construct the PPI network, and then Cytoscape software was used to visualize the distribution characteristics of the top 10 up- and downregulated DEGs in the PPI network [18].

\section{Results}

3.1. Short Overview of the Studies Included. In this study, we obtained a total of 5 expression profiles of pituitary adenoma in GEO database; it contained 44 samples of pituitary adenoma and 12 samples of controls. The individual studies for analyzing are displayed in Table 1. Several types of pituitary adenomas were included in our study such as NFPA, growth hormone pituitary adenomas, and prolactin adenomas.

3.2. Detecting Genes Associated with Pituitary Adenoma. After global normalization, we adopted SAM software to identify DEGs between pituitary adenomas and control samples. With FDR $\leq 0.05$ and a minimal fold change of 1.5, a total of 3994 genes were found to show aberrant expression in samples of pituitary adenoma compared with NC tissues, among which 2043 DEGs were upregulated and 1951 were downregulated. A list of the top 10 most significantly up- or downregulated genes was presented in Table 2.

The upregulated gene with the lowest $P$ value was C7orf62, whose function has been unclear. The downregulated gene with the lowest $P$ value was $R D H 10$, which is essential for synthesis of embryonic retinoic acid and limb, craniofacial, and organ development. The full list of these genes was 
TABle 2: The top 10 most significantly up- or down-regulated DEGs.

\begin{tabular}{|c|c|c|c|c|}
\hline Gene ID & Gene symbol & Official full name & $P$ value & Fold change \\
\hline \multicolumn{5}{|c|}{ Up-regulated genes } \\
\hline 219557 & C7orf62 & Chromosome 7 open reading frame 62 & $3.33 E-16$ & 2.1819 \\
\hline 57519 & STARD9 & StAR-related lipid transfer (START) domain containing 9 & $1.28 E-14$ & 1.8589 \\
\hline 89792 & GAL3ST3 & Galactose-3-O-sulfotransferase 3 & $1.29 E-13$ & 1.9935 \\
\hline 57650 & KIAA1524 & KIAA1524 & $1.09 E-12$ & 1.351 \\
\hline 114757 & CYGB & Cytoglobin & $4.85 E-12$ & 1.8788 \\
\hline 157983 & C9orf66 & Chromosome 9 open reading frame 66 & $1.73 E-11$ & 1.4951 \\
\hline 341880 & SLC35F4 & Solute carrier family 35 , member F4 & $1.91 E-11$ & 2.2741 \\
\hline 57121 & LPAR5 & Lysophosphatidic acid receptor 5 & $3.26 E-11$ & 1.3792 \\
\hline 257044 & Clorf101 & Chromosome 1 open reading frame 101 & $3.63 E-11$ & 1.2287 \\
\hline 145581 & LRFN5 & Leucine rich repeat and fibronectin type III domain containing 5 & $3.80 E-11$ & 1.9332 \\
\hline \multicolumn{5}{|c|}{ Down-regulated genes } \\
\hline 157506 & RDH10 & Retinol dehydrogenase 10 (all-trans) & 0 & -2.3698 \\
\hline 8324 & FZD7 & Frizzled class receptor 7 & 0 & -2.6494 \\
\hline 140576 & S100A16 & S100 calcium binding protein A16 & $3.00 E-15$ & -1.8248 \\
\hline 84952 & CGNL1 & Cingulin-like 1 & $2.40 E-14$ & -2.1091 \\
\hline 85375 & KIAA1661 & KIAA1661 protein & $4.95 E-14$ & -1.896 \\
\hline 55276 & PGM2 & Phosphoglucomutase 2 & $7.29 E-14$ & -1.5698 \\
\hline 55300 & PI4K2B & Phosphatidylinositol 4-kinase type 2 beta & $9.64 E-14$ & -1.4987 \\
\hline 84899 & TMTC4 & Transmembrane and tetratricopeptide repeat containing 4 & $1.20 E-13$ & -1.4509 \\
\hline 345557 & PLCXD3 & Phosphatidylinositol-specific phospholipase $\mathrm{C}, \mathrm{X}$ domain containing 3 & $3.01 E-13$ & -3.3246 \\
\hline 5570 & PKIB & Protein kinase (cAMP-dependent, catalytic) inhibitor beta & $1.00 E-12$ & -2.3052 \\
\hline
\end{tabular}

provided as Supplementary Table 1 in Supplementary Material available online at http://dx.doi.org/10.1155/2015/164087.

3.3. Functional Annotation. To understand the biological roles of the DEGs from pituitary adenomas, we conducted GO categories and KEGG pathway enrichment analysis. GO categories are separated into three groups: biological process, cellular component, and molecular function. We examined GO categories separately. The significantly enriched GO terms for molecular functions were carbohydrate binding (GO: $0030246, P=1.93 E-03$ ) and calcium ion binding (GO: 0005509, $P=3.10 E-03$ ) for molecular functions, while for biological processes they were aminoglycan metabolic process (GO: 0006022, $P=9.76 E-04$ ) and sulfur metabolic process (GO: 0006790, $P=1.07 E-03$ ), and for cellular component they were intrinsic to membrane (GO: 0031224, $P=2.76 E-04$ ) and integral to membrane (GO: 0016021, $P=7.43 E-04)$ (Figure 1).

Hypergeometric test with $P$ value $<0.05$ was used as the criteria for pathway detection (Table 3 ). The most significant pathway in our analysis was neuroactive ligand-receptor interaction $(P=3.97 E-03)$. Furthermore, tryptophan metabolism $(P=2.42 E-02)$ and cardiac muscle contraction $(P=5.38 E-02)$ are also highly enriched.

3.4. Protein-Protein Interaction (PPI) Network Construction. The PPI networks of the top 10 upregulated and downregulated DEGs were established by Cytoscape software including
TABLE 3: The enriched KEGG pathway of DEGs.

\begin{tabular}{lcc}
\hline KEGG pathway & $\begin{array}{c}\text { Number of } \\
\text { genes }\end{array}$ & $P$ value \\
\hline $\begin{array}{l}\text { Neuroactive ligand-receptor } \\
\text { interaction }\end{array}$ & 54 & $3.97 E-03$ \\
$\begin{array}{l}\text { Tryptophan metabolism } \\
\text { Cardiac muscle contraction }\end{array}$ & 12 & $2.42 E-02$ \\
O-Glycan biosynthesis & 9 & $5.38 E-02$ \\
Taste transduction & 13 & $6.01 E-02$ \\
TGF-beta signaling pathway & 19 & $7.43 E-02$ \\
$\begin{array}{l}\text { Arrhythmogenic right ventricular } \\
\text { cardiomyopathy (ARVC) }\end{array}$ & 17 & $7.83 E-02$ \\
Basal cell carcinoma & 13 & $9.46 E-02$ \\
Colorectal cancer & 18 & $9.56 E-02$ \\
$\begin{array}{l}\text { Amino sugar and nucleotide sugar } \\
\text { metabolism }\end{array}$ & 11 & $9.71 E-02$ \\
\hline
\end{tabular}

77 nodes, 76 edges. In the PPI network the nodes with high degree are defined as hub protein, and degrees are defined to measure how many neighbors a node directly connects to. The significant hub proteins in our PPI networks contained S100A16 $($ Degree $=27)$, PKIB $($ Degree $=8$ ), and PGM2 (Degree $=7$ ) (Figure 2 ) 


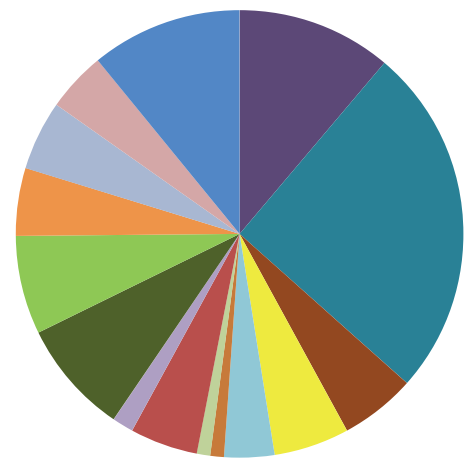

- Carbohydrate binding [80]

- Calcium ion binding [182]

- Polysaccharide binding [39]

- Pattern binding [39]

- Serine-type endopeptidase inhibitor activity [26]

- Rhodopsin-like receptor activity [7]

- Vitamin transporter activity [7]

- Glycosaminoglycan binding [35]

- RNA polymerase II transcription mediator activity [11]

- Enzyme inhibitor activity [59]

- Alkali metal ion binding [51]

- Endopeptidase inhibitor activity [35]

- Peptidase inhibitor activity [36]

- Potassium ion binding [31]

- Guanyl ribonucleotide binding [78]

(a)

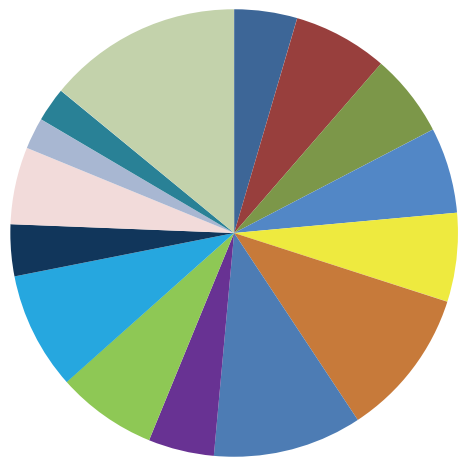

Aminoglycan metabolic process [22]

Sulfur metabolic process [33]

- Sensory perception of sound [29]

- Sensory perception of mechanical stimulus [30]

- Polysaccharide metabolic process [31]

- Sensory perception of light stimulus [52]

Visual perception [52]

- Vitamin metabolic process [23]

- Embryonic organ morphogenesis [35]

- Extracellular structure organization [41]

- Glycosaminoglycan metabolic process [18]

1. Biogenic amine metabolic process [27]

- Apoptotic nuclear changes [11]

- Biogenic amine biosynthetic process [12]

- Embryonic morphogenesis [68]

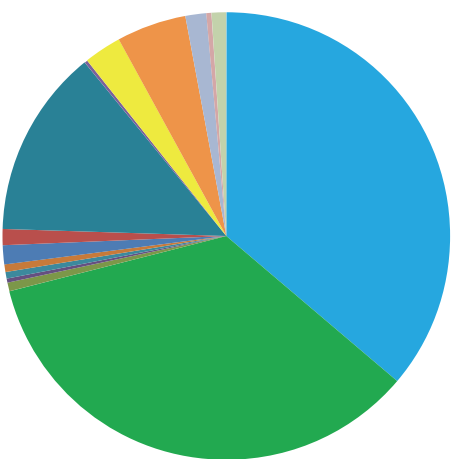

- Intrinsic to membrane [987]

- Integral to membrane [950]

- Intrinsic to Golgi membrane [17]

- Intercalated disc [8]

- Spindle pole [13]

Integral to Golgi membrane [15]

- Spindle [37]

- Chromosome, centromeric region [32]

- Extracellular region [371]

- Fascia adherens [6]

- Extracellular matrix [74]

- Cell projection [137]

- Apical part of cell [41]

- Spindle microtubule [10]

Integral to organelle membrane [29]

(b)

(c)

FIgure 1: The top 15 enriched GO terms of DEGs. (a) Molecular functions for DEGs ( $P$ value $\leq 4.61 E-03)$; (b) biological process for DEGs $(P$ value $\leq 6.54 E-03)$; (c) cellular component for DEGs $(P$ value $\leq 1.95 E-03)$.
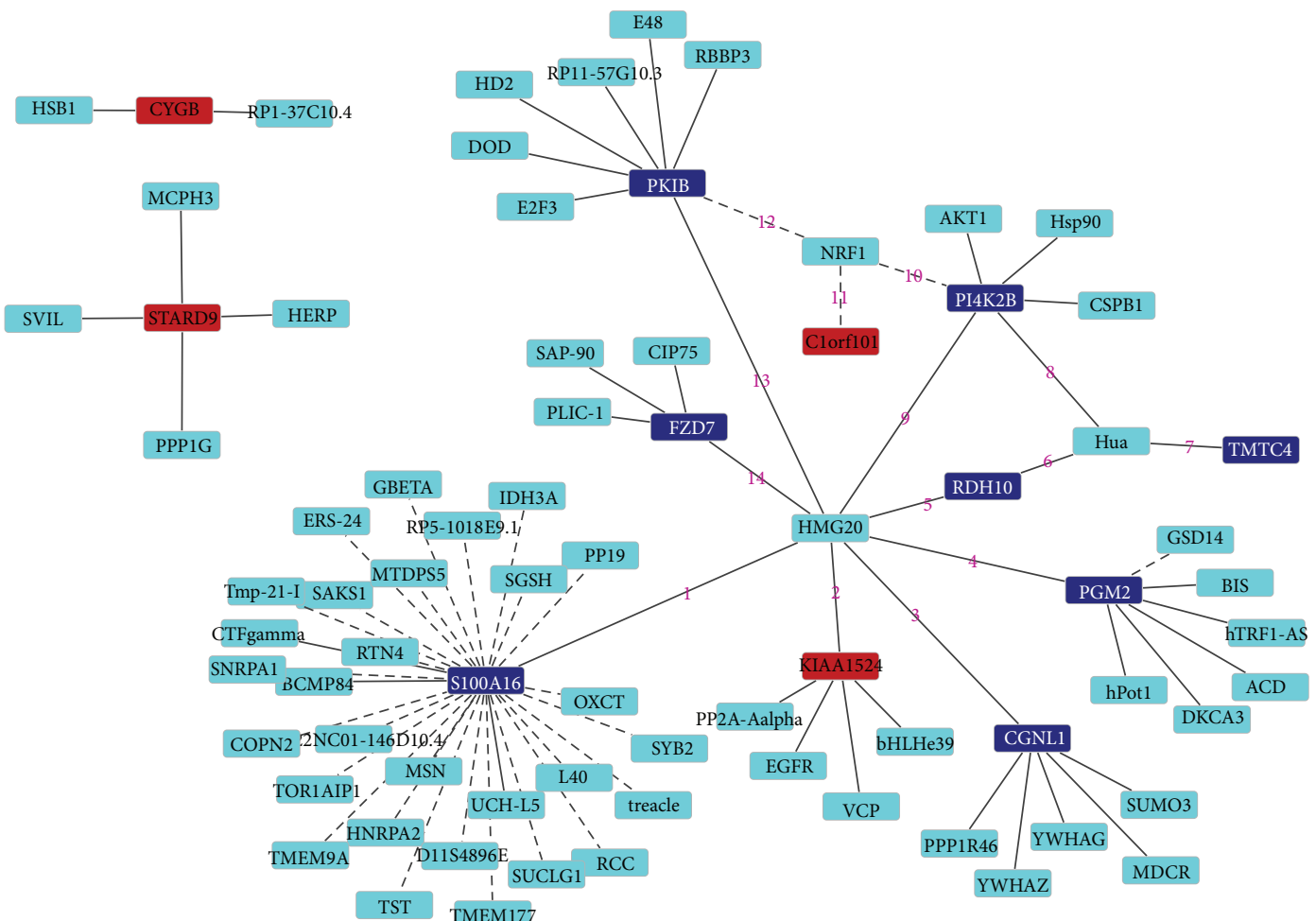

FIgURE 2: The constructed PPI network for the top 10 up- and downregulated DEGs. Nodes represent proteins; edges represent interactions between two proteins. Red- and blue-color nodes represent products of up- and downregulated DEGs, respectively. Green nodes denote products of genes predicted to interact with the DEGs. 


\section{Discussion}

Pituitary adenomas are monoclonal in origin and most of them are benign, slow-growing neoplasms, and so it is acceptable that many common oncogenes or tumor-suppressor genes which have been confirmed to be involved in human cancers have been indiscoverable in pituitary adenomas. However, advances in molecular biology make it possible to fast-track the search for candidate tumourigenic genes of pituitary adenomas. Some researchers have compared gene expression profiling and identified DEGs between normal human pituitary and pituitary adenomas of different cell origins via microarray technique. Previous microarray studies of human pituitary adenomas have detected many novel candidate genes, including PTTG [19], GADD45 [20], MEG3a [21], and BMP-4 [22].

In this paper, we chose an integrated analysis approach by which 5 microarray datasets were combined to highlight genes that were consistently expressed differentially with statistical significance, performed GO and KEGG pathway enrichment analysis for these genes, and finally constructed PPI networks of the top 10 upregulated and downregulated DEGs. In our integrated analysis, a total of 3994 genes were found to show altered expression in samples of pituitary adenoma compared with NC tissues (2043 upregulated and 1951 downregulated genes). The upregulated gene with the lowest $P$ value was $C 7$ orf62, whose function has been unclear. The downregulated gene with the lowest $P$ value was $R D H 10$, a member of short chain dehydrogenase-reductase (SDR) family, which is essential for synthesis of embryonic retinoic acid. Additionally $\mathrm{RDH} 10$ was reported to be necessary for limb, craniofacial, and organ development by inducing proliferation arrest, differentiation, and apoptosis [23, 24]. Bankovic et al. found that patients of non-small-cell lung cancer with mutated $\mathrm{RDH} 10$ had shorter survival than those without mutated RDH10, confirming its importance in tumor progression. Previous study also identified that $R D H 10$ played an important role in tumors with lymph node invasion [25]. The role and association with pituitary adenoma have not yet been reported.

In line with previous findings, some genes identified in our integrated analysis have been closely related to the tumorigenesis of pituitary adenomas, such as GADD45G, GADD45B, MEG3 [26], POU1F1 [27], IGFBP3 [28], and CCNB1 [29]. GADD45B and GADD45G belong to GADD45 gene family, and loss of GADD45 expression has been observed in various human cancers [30]. It has been proved that loss of or significantly reduced GADD45G expression is found in most of the pituitary adenomas due to promoter methylation [31]. GADD45B was displayed to be downregulated in pituitary adenomas (-68-fold) through microarray data, subsequently verified by qPCR and immunoblotting, and in vitro experiments identified its role as a tumor suppressor [32]. Cheunsuchon et al. indicated that MEG3 was specifically lost in NFAs, suggesting that its inactivation might be involved in the development of NFAs [33]. The other genes including $P O U 1 F 1, I G F B P 3$, and $C C N B 1$ were also reported to correlate with pituitary adenomas.
In order to reveal the biological roles of the DEGs from pituitary adenoma, we performed functional annotation for these genes. Neuroactive ligand-receptor interaction, tryptophan metabolism, and cardiac muscle contraction are found to be the top 3 significantly enriched pathways. Many signal transduction pathways are involved in the development of pituitary adenoma including TGF- $\beta$ signal pathway, Wnt signal pathway, and MAPK signal pathway. Interestingly, we noted that the most significant pathway in our analysis was neuroactive ligand-receptor interaction, which is physiologically associated with the neuronal functions. The neuroactive ligand-receptor interaction pathway, which is a collection of neuroactive receptors located on the plasma membranes, is implicated in the stabilization of the neuroendocrine system, indicating that neuroactive ligand-receptor interaction pathway may be involved in the development of pituitary adenoma $[34,35]$.

Furthermore PPI networks of the top 10 upregulated and downregulated DEGs indicated that the significant hub proteins contained S100A16, PKIB, and PGM2. S100A16, a novel member of S100 protein family which is implicated in cognition in the central nervous system [36], is closely associated with brain pathologies [37]. S100A16 has also been shown to be ubiquitously expressed and upregulated in human tumor [38]. However its function in pituitary adenoma is still unknown for lack of related studies.

The present study has several limitations. First, although global normalization was performed to minimize the heterogeneity of various microarray studies, the heterogeneity cannot be removed completely, which may have distorted the result of analysis. Second, due to limited microarray studies in pituitary adenomas, different subtypes of pituitary adenomas were analyzed, which was not taken into account in our study. Despite these limitations, our findings have important implications for the molecular mechanisms of pituitary adenoma, adding new insights into the future therapy.

In conclusion, by the integrated analysis we have shown the underlying molecular differences between the normal human pituitary and pituitary adenomas of different cell origins and identified DEGs and biological function to contribute to the successful identification of therapeutic targets for pituitary adenoma and the development of effective targeted therapies. Further functional studies may provide additional insights into the role of the differentially regulated genes in the pathophysiology of pituitary adenoma.

\section{Conflict of Interests}

The authors declare that they have no conflict of interests.

\section{Acknowledgments}

This study was supported by the National Natural Science Fund of China (No. 30900342), the Research Special Fund for Public Welfare Industry of Health (No. 201402008) and the National High Technology Research and Development Program (“863”Program) of China. 


\section{References}

[1] S. L. Asa and S. Ezzat, "The cytogenesis and pathogenesis of pituitary adenomas," Endocrine Reviews, vol. 19, no. 6, pp. 798$827,1998$.

[2] N. H. Lee and A. I. Saeed, "Microarrays," in Protocols for Nucleic Acid Analysis by Nonradioactive Probes, pp. 265-300, Springer, New York, NY, USA, 2007.

[3] M. S. Elston, A. J. Gill, J. V. Conaglen et al., "Wnt pathway inhibitors are strongly down-regulated in pituitary tumors," Endocrinology, vol. 149, no. 3, pp. 1235-1242, 2008.

[4] C.-O. Evans, P. Reddy, D. J. Brat et al., "Differential expression of folate receptor in pituitary adenomas," Cancer Research, vol. 63, no. 14, pp. 4218-4224, 2003.

[5] D. G. Morris, M. Muşat, S. Czirják et al., "Differential gene expression in pituitary adenomas by oligonucleotide array analysis," European Journal of Endocrinology, vol. 153, no. 1, pp. 143-151, 2005.

[6] K. H. Ruebel, A. A. Leontovich, L. Jin et al., "Patterns of gene expression in pituitary carcinomas and adenomas analyzed by high-density oligonucleotide arrays, reverse transcriptasequantitative PCR, and protein expression," Endocrine, vol. 29, no. 3, pp. 435-444, 2006.

[7] A. S. Siddiqui, A. D. Delaney, A. Schnerch, O. L. Griffith, S. J. M. Jones, and M. A. Marra, "Sequence biases in large scale gene expression profiling data," Nucleic Acids Research, vol. 34, no. 12, article e83, 2006.

[8] J. Feichtinger, G. G. Thallinger, R. J. McFarlane, and L. D. Larcombe, "Microarray meta-analysis: from data to expression to biological relationships," in Computational Medicine, pp. 5977, Springer, New York, NY, USA, 2012.

[9] A. Ramasamy, A. Mondry, C. C. Holmes, and D. G. Altman, "Key issues in conducting a meta-analysis of gene expression microarray datasets," PLoS Medicine, vol. 5, no. 9, article e184, 2008.

[10] Y. Tong, Y. Zheng, J. Zhou, N. M. Oyesiku, H. P. Koeffler, and S. Melmed, "Genomic characterization of human and rat prolactinomas," Endocrinology, vol. 153, no. 8, pp. 3679-3691, 2012.

[11] A. Wierinckx, M. Roche, G. Raverot et al., "Integrated genomic profiling identifies loss of chromosome 11p impacting transcriptomic activity in aggressive pituitary PRL tumors," Brain Pathology, vol. 21, no. 5, pp. 533-543, 2011.

[12] T. Barrett, S. E. Wilhite, P. Ledoux et al., "NCBI GEO: archive for functional genomics data sets-update," Nucleic Acids Research, vol. 41, no. 1, pp. D991-D995, 2013.

[13] V. G. Tusher, R. Tibshirani, and G. Chu, "Significance analysis of microarrays applied to the ionizing radiation response," Proceedings of the National Academy of Sciences of the United States of America, vol. 98, no. 9, pp. 5116-5121, 2001.

[14] E. Altermann and T. R. Klaenhammer, "PathwayVoyager: pathway mapping using the Kyoto Encyclopedia of Genes and Genomes (KEGG) database," BMC Genomics, vol. 6, article 60, 2005.

[15] D. Tabas-Madrid, R. Nogales-Cadenas, and A. PascualMontano, "GeneCodis3: a non-redundant and modular enrichment analysis tool for functional genomics," Nucleic Acids Research, vol. 40, no. 1, pp. W478-W483, 2012.

[16] L. Giot, J. S. Bader, C. Brouwer et al., "A protein interaction map of Drosophila melanogaster," Science, vol. 302, no. 5651, pp. 17271736, 2003.
[17] S. Li, C. M. Armstrong, N. Bertin et al., "A map of the interactome network of the metazoan C. elegans," Science, vol. 303, no. 5657, pp. 540-543, 2004.

[18] P. Shannon, A. Markiel, O. Ozier et al., "Cytoscape: a software environment for integrated models of biomolecular interaction networks," Genome Research, vol. 13, no. 11, pp. 2498-2504, 2003.

[19] L. Pei and S. Melmed, "Isolation and characterization of a pituitary tumor-transforming gene (PTTG)," Molecular Endocrinology, vol. 11, no. 4, pp. 433-441, 1997.

[20] A. Bahar, J. E. Bicknell, D. J. Simpson, R. N. Clayton, and W. E. Farrell, "Loss of expression of the growth inhibitory gene GADD $45 \gamma$, in human pituitary adenomas, is associated with CpG island methylation," Oncogene, vol. 23, no. 4, pp. 936-944, 2004.

[21] X. Zhang, Y. Zhou, K. R. Mehta et al., "A pituitary-derived MEG3 isoform functions as a growth suppressor in tumor cells," The Journal of Clinical Endocrinology \& Metabolism, vol. 88, no. 11, pp. 5119-5126, 2003.

[22] M. Páez-Pereda, D. Giacomini, D. Refojo et al., "Involvement of bone morphogenetic protein 4 (BMP-4) in pituitary prolactinoma pathogenesis through a Smad/estrogen receptor crosstalk," Proceedings of the National Academy of Sciences of the United States of America, vol. 100, no. 3, pp. 1034-1039, 2003.

[23] B. X. Wu, Y. Chen, J. Fan, B. Rohrer, R. K. Crouch, and J.-X. Ma, "Cloning and characterization of a novel all-trans retinol shortchain dehydrogenase/reductase from the RPE," Investigative Ophthalmology and Visual Science, vol. 43, no. 11, pp. 3365-3372, 2002.

[24] P. Picozzi, A. Marozzi, D. Fornasari et al., "Genomic organization and transcription of the human retinol dehydrogenase 10 (RDH10) gene," FEBS Letters, vol. 554, no. 1-2, pp. 59-66, 2003.

[25] J. Bankovic, J. Stojsic, D. Jovanovic et al., "Identification of genes associated with non-small-cell lung cancer promotion and progression," Lung Cancer, vol. 67, no. 2, pp. 151-159, 2010.

[26] Y. Zhou, X. Zhang, and A. Klibanski, "Genetic and epigenetic mutations of tumor suppressive genes in sporadic pituitary adenoma," Molecular and Cellular Endocrinology, vol. 386, no. 1-2, pp. 16-33, 2014.

[27] R. V. Araujo, C. V. Chang, V. A. S. Cescato et al., "PROP1 overexpression in corticotrophinomas: evidence for the role of PROP1 in the maintenance of cells committed to corticotrophic differentiation," Clinics, vol. 68, no. 6, pp. 887-891, 2013.

[28] Z.-Q. Jiang, S.-B. Gui, and Y.-Z. Zhang, "Differential gene expression by fiber-optic beadarray and pathway in adrenocorticotrophin-secreting pituitary adenomas," Chinese Medical Journal, vol. 123, no. 23, pp. 3455-3461, 2010.

[29] Y. Tani, N. Inoshita, T. Sugiyama et al., "Upregulation of CDKN2A and suppression of cyclin D1 gene expressions in ACTH-secreting pituitary adenomas," European Journal of Endocrinology, vol. 163, no. 4, pp. 523-529, 2010.

[30] R. E. Tamura, J. F. de Vasconcellos, D. Sarkar, T. A. Libermann, P. B. Fisher, and L. F. Zerbini, "GADD45 proteins: central players in tumorigenesis," Current Molecular Medicine, vol. 12, no. 5, pp. 634-651, 2012.

[31] A. Bahar, J. E. Bicknell, D. J. Simpson, R. N. Clayton, and W. E. Farrell, "Loss of expression of the growth inhibitory gene GADD $45 \gamma$, in human pituitary adenomas, is associated with CpG island methylation," Oncogene, vol. 23, no. 4, pp. 936-944, 2004.

[32] K. A. Michaelis, A. J. Knox, M. Xu et al., "Identification of growth arrest and DNA-damage-inducible gene beta 
(GADD45beta) as a novel tumor suppressor in pituitary gonadotrope tumors," Endocrinology, vol. 152, no. 10, pp. 36033613, 2011.

[33] P. Cheunsuchon, Y. Zhou, X. Zhang et al., "Silencing of the imprinted DLK1-MEG3 locus in human clinically nonfunctioning pituitary adenomas," The American Journal of Pathology, vol. 179, no. 4, pp. 2120-2130, 2011.

[34] M. Lauss, A. Kriegner, K. Vierlinger, and C. Neohammer, "Characterization of the drugged human genome," Pharmacogenomics, vol. 8, no. 8, pp. 1063-1073, 2007.

[35] F. Wang, W. Liu, Y. Jin et al., "Transcriptional effects of prenatal and neonatal exposure to PFOS in developing rat brain," Environmental Science and Technology, vol. 44, no. 5, pp. 18471853, 2010.

[36] L. J. Van Eldik and M. S. Wainwright, "The Janus face of glial-derived S100B: beneficial and detrimental functions in the brain," Restorative Neurology and Neuroscience, vol. 21, no. 3-4, pp. 97-108, 2003.

[37] D. R. Ziegler, C. E. Innocente, R. B. Leal, R. Rodnight, and C. A. Gonçalves, "The S100B protein inhibits phosphorylation of GFAP and vimentin in a cytoskeletal fraction from immature rat hippocampus," Neurochemical Research, vol. 23, no. 10, pp. 1259-1263, 1998.

[38] I. Marenholz and C. W. Heizmann, "S100A16, a ubiquitously expressed EF-hand protein which is up-regulated in tumors," Biochemical and Biophysical Research Communications, vol. 313, no. 2, pp. 237-244, 2004. 


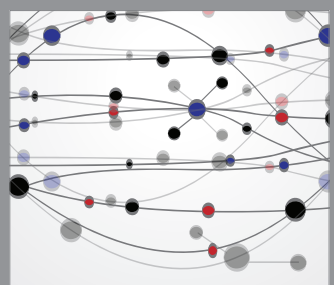

The Scientific World Journal
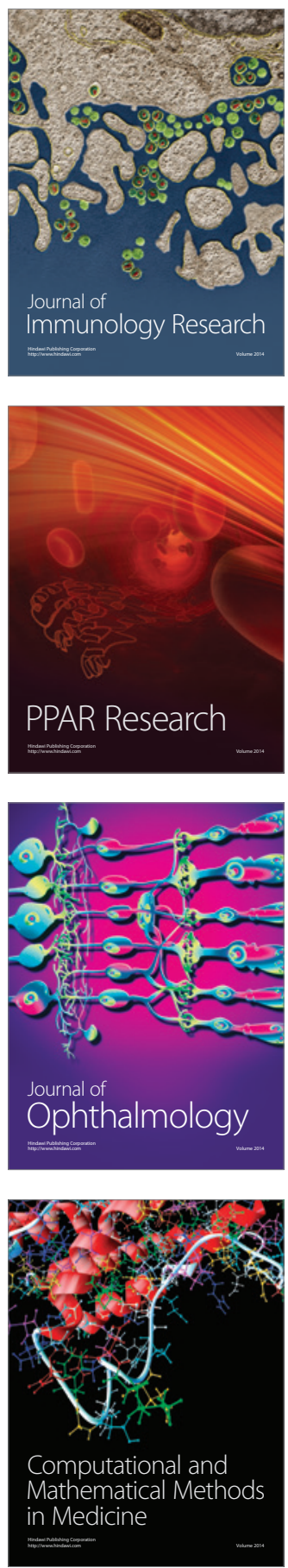

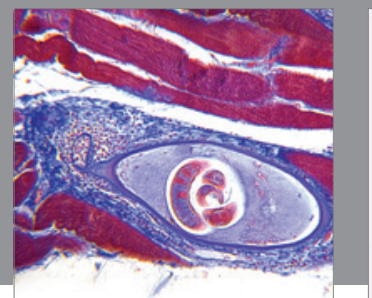

Gastroenterology

Research and Practice
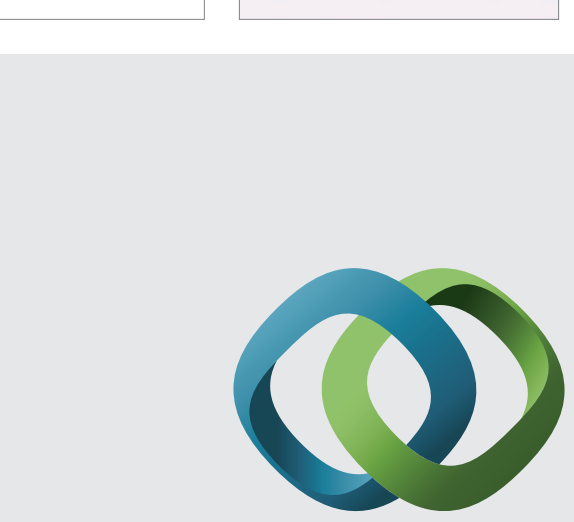

\section{Hindawi}

Submit your manuscripts at

http://www.hindawi.com
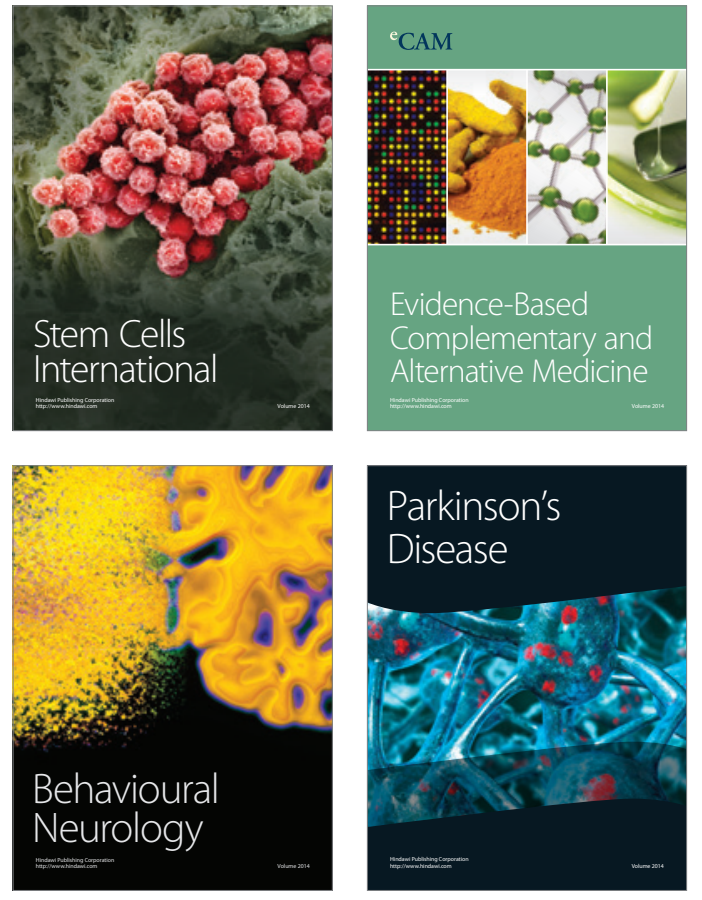
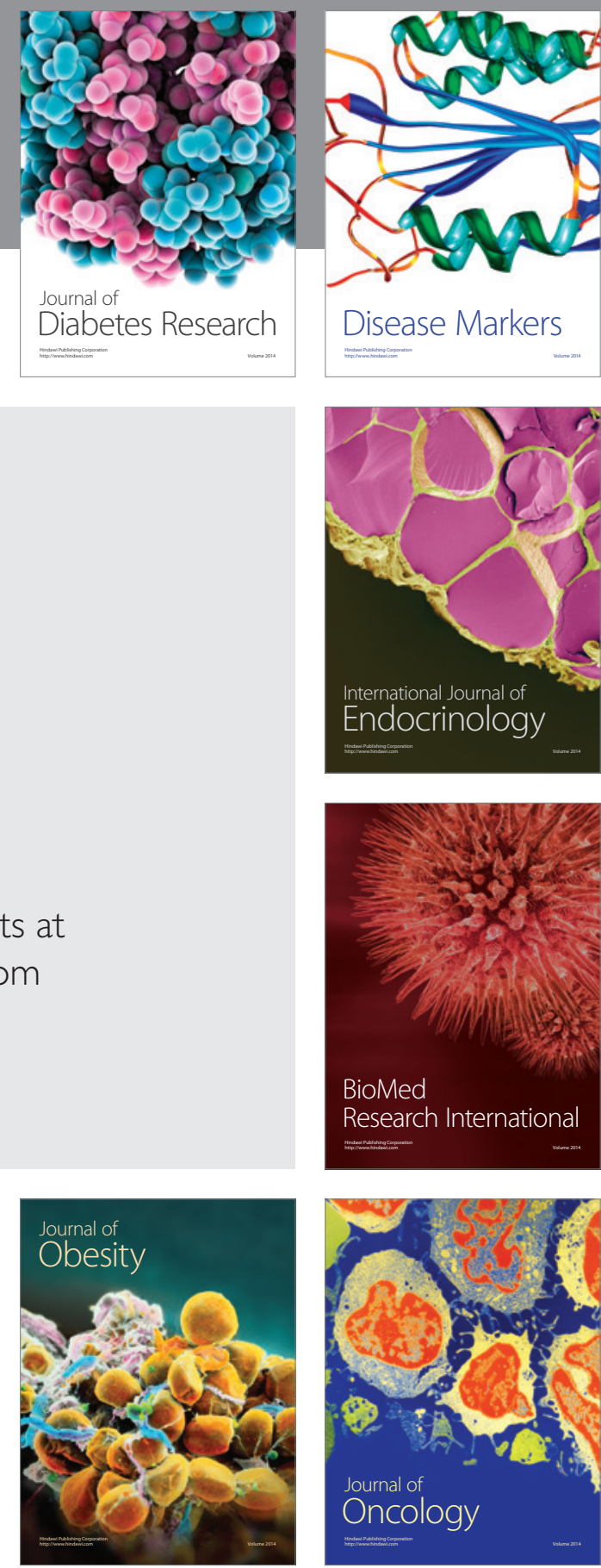

Disease Markers
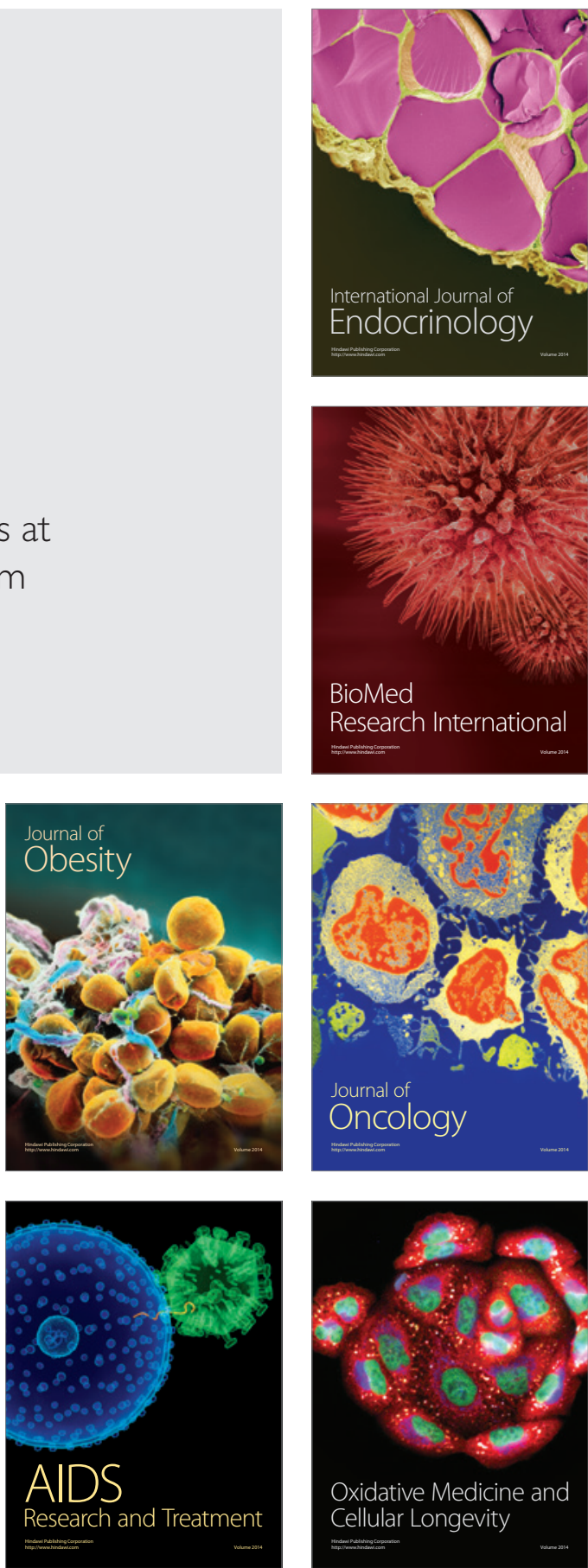